\title{
VALORES RELATIVOS AO TRABALHO EM UMA INSTITUIÇÃO PÚBLICA DE ENSINO SUPERIOR
}

\author{
WORK VALUES IN A FEDERAL EDUCATIONAL INSTITUTION
}

Simone Alves Pacheco de Campos

Doutoranda em Administração na Universidade Federal do Rio Grande do Sul

Porto Alegre, RS, Brasil

E-mail: simoneapcampos@gmail.com

Eliete dos Reis Lehnhart

Doutoranda em Administração na Universidade Federal de Santa Maria

Santa Maria, RS, Brasil

E-mail: elietedosreis@gmail.com

Maria Ivete Trevisan Fossá

Professora na Universidade Federal de Santa Maria

Santa Maria, RS, Brasil

E-mail: fossa@terra.com.br

Laércio André Gassen Balsan

Doutorando em Administração na Universidade Federal de Santa Maria

Santa Maria, RS, Brasil

E-mail: laerciobalsan@yahoo.com.br

\section{RESUMO}

Este estudo teve como objetivo identificar como se estrutura a hierarquia dos valores relativos ao trabalho em uma instituição pública de ensino superior. Para tanto, realizou-se uma pesquisa descritiva, de caráter quantitativo, cujos resultados demonstraram que os valores se encontram hierarquizados segundo a seguinte estrutura: Estabilidade e Realização no Trabalho, Relações Sociais e Prestígio. As mulheres diferiram dos homens em relação à ênfase na busca por realização e estabilidade. Indivíduos com idade superior à idade média buscam sucesso profissional e poder de influência, concomitantemente com a manutenção de relações sociais positivas no trabalho. Servidores com mais tempo de cargo buscam a realização no trabalho. Por fim, a realização desta pesquisa forneceu importantes contribuições à gestão considerando que, a partir da compreensão dos valores relativos ao trabalho as organizações podem direcionar seus esforços e práticas à manutenção de um ambiente de trabalho voltado à realização das metas dos seus funcionários.

Palavras-chave: Gestão de Recursos Humanos. Valores Relativos ao Trabalho. Valores Humanos. Valores Pessoais. Administração Pública.

Data de submissão: 26 de novembro de 2014.

\section{ABSTRACT}

This study aims to identify work values' hierarchical structure in a public university. Therefore, we carried out a descriptive and quantitative research. It was observed that the values are prioritized according to the following structure: Stability and Performance at Work, Social Relations and prestige. Employees older than the average age seek professional success and power of influence, concurrently with the maintenance of positive social relationships at work. Employees with tenure higher tend to value the pursuit of fulfillment at work. This research provided important contributions to management whereas, from the understanding of work values organizations can target their efforts and practices to maintain a work environment focused on the employees' goal achievement.

Keywords: Human Resource Management. Work Values. Human Values. Personal Values. Public Administration.

Data de aprovação: 20 de julho de 2016 . 


\section{INTRODUÇÃO}

O trabalho tem um papel central na vida dos indivíduos (MORIN, 2002; PORTO e TAMAYO, 2008), não somente como forma de sustento, mas também, por ser considerado a base da participação e status social (ROE e ESTER, 1999).

Dentro desse contexto, estudos têm contribuído de forma significativa para a compreensão das maneiras pelas quais os tipos motivacionais de valores estão relacionados a comportamentos, experiências e papéis sociais (ROS, SCHWARTZ e SURKISS, 1999; TAMAYO, 2001), fornecendo valiosos insights acerca das mudanças da sociedade, do comportamento das pessoas e das diferenças entre grupos (PORTO e TAMAYO, 2003).

No setor público, essa temática assume grande importância, principalmente pela imagem que tem sido atribuída ao servidor público, como a "ineficiência decorrente de um excessivo aparato burocrático e o baixo engajamento de seus dirigentes e servidores com o propósito maior dessas mesmas organizações" (MORAES, MARQUES e CORREIA, 1998, p.2). A construção dessa imagem social tem como condicionante o padrão de conduta do servidor, que por vez, deve ser entendido dentro do contexto social, político e econômico (BRANDÃO e BASTOS, 1993).

No caso de Universidades Públicas, tão questionadas quanto ao seu desempenho e ao seu papel social (BRANDÃO e BASTOS, 1993), a compreensão dos valores relativos ao trabalho assume relevância ainda maior. A identificação das prioridades axiológicas de seus servidores possibilita a criação de estratégias e ambientes de trabalho que permitirão a satisfação de suas crenças, gerando assim, uma melhoria dos resultados organizacionais: prestação de serviços educacionais de qualidade. Sobretudo, compreender a motivação para o trabalho no serviço público é refletir sobre as questões que afastam o indivíduo da iniciativa privada, levando em consideração os aspectos que os tornam trabalhadores distintos.

Diante desse contexto, emerge a seguinte inquietação de pesquisa: "Como se estruturam hierarquicamente os valores relativos ao trabalho de servidores de uma instituição pública de Ensino Superior"?

Assim, a fim de contribuir para a exploração do tema, neste estudo objetiva-se: (i) identificar a estrutura hierárquica de valores relativos ao trabalho numa Instituição Pública de Ensino Superior; e (ii) verificar se a hierarquia de valores relativos ao trabalho difere em relação às variáveis pessoais e organizacionais.

\section{VALORES HUMANOS: ENTENDENDO A BASE DOS VALORES PESSOAIS}

Os valores têm sido alvo de estudos para a compreensão e explicação do comportamento do indivíduo, das mudanças ocorridas na sociedade, para julgar ações, bem como diferenciar nações e grupos (TAMAYO e PORTO, 2005). O estudo dos valores humanos teve início na filosofia, principalmente nos estudos dos filósofos Spranger, em 1928, e, após por Alport e Vernom, em 1931 (BILSKY, 2009, TAMAYO et al., 1998; ROHAN, 2000). Nessa linha, Almeida e Sobral (2009) afirmam que o interesse pelos valores, por parte da psicologia, desenvolveu-se a partir da segunda metade do século $X X$, fazendo com que as teorias $\mathrm{e}$ pesquisas de campo fossem projetadas para além do escopo da reflexão filosófica, a partir do 
desenvolvimento de maneiras de operacionalização do conceito e ampliando-se no campo dos estudos empíricos.

Almeida e Sobral (2009), afirmam que durante as últimas décadas, a terminologia dos valores foi adotada em diversas formas no campo dos estudos organizacionais, além daquelas mais comuns utilizadas na linguagem econômica. Esses mesmos autores enfatizam que os valores passaram a contribuir para o entendimento da cultura organizacional, de princípios que levam a ações, bem como para explicar o comportamento dos indivíduos em contextos organizacionais. O sistema de valores de um indivíduo está presente em diversas facetas de sua vida, manifestando-se em suas escolhas e na forma como se relaciona com os outros (SCHWARTZ, 2005).

Rokeach (1973) conceitua valores como padrões ou critérios que servem a importantes propósitos na vida cotidiana do indivíduo, sendo utilizados como critérios para orientar e julgar seus comportamentos e o comportamento dos outros. Assim, valores transcendem objetivos e situações específicas, relacionam-se com modos de condutas ou estados-fim de existência, intransferíveis, pessoal e socialmente preferíveis a outros modos de conduta ou estados-fins de existência alternativos (ROKEACH, 1973).

Em sua pesquisa, o autor desenvolveu o inventário Rokeach Value Survey (RVS) com objetivo de identificar a prioridade de cada valor na vida da pessoa criando uma lista de 18 valores terminais (metas de vida) e 18 valores instrumentais (formas de condutas). Sua teoria representou uma grande influencia para a psicologia social, muito embora atualmente tenha sido questionada por autores de outras disciplinas (SCHWARTZ e BILSKY. 1987; BILSKY 2009, LYONS, DUXBURRY e HIGGINS, 2010).

A teoria de valores, de acordo com o entendimento de Schwartz (1992), descreve aspectos fundamentais da estrutura psicológica do indivíduo, os quais são presumivelmente comuns a todos os seres humanos. Tamayo e Gondim (1996) acrescentam que pesquisas como de Schwartz (1992, 1994), Tamayo e Schwartz (1993) revelaram que, em geral, os valores humanos apresentam uma estrutura baseada nas metas perseguidas pelos indivíduos. Desse modo, valores identificados e priorizados pelos indivíduos são importantes e auxiliam na compreensão do pensamento e das ações humanas (PORTO e TAMAYO, 2005).

Para Schwartz e Bilsky (1987), os valores podem ser entendidos como crenças ou princípios, acerca de comportamentos ou estados de existência, que transcendem situações específicas, guiam a seleção de comportamentos e eventos e são ordenados por sua importância relativa aos demais. A definição proposta pelos autores baseia-se no entendimento de que os valores são representações cognitivas de três exigências humanas universais: necessidades biológicas, necessidades de interação e necessidades sócio-institucionais de sobrevivência e bem-estar dos grupos (SCHWARTZ e BILSKY, 1987).

Dessa forma, segundo Porto e Tamayo (2003, p. 146), "as pessoas apresentam uma estrutura de valores que exerce uma função motivacional importante e que está relacionada com os aspectos fundamentais de sua vida". A estrutura, ou sistema de valores, para esse autor, pode ser compreendida a partir de duas dimensões: a primeira, evidenciada nesta seção, refere-se a estrutura geral de valores, relacionada a todas as partes da vida; e, a segunda, expressa na próxima seção, trata de estruturas de valores referentes a determinados contextos específicos.

Considerando a estrutura geral dos valores, cabe acrescentar a teoria de valores básicos proposta por Schwartz (1992, 1994), a qual baliza os trabalhos desenvolvidos acerca dessa temática. Essa teoria propõe uma estrutura de dez tipos motivacionais distintos, de acordo com a motivação subjacente a cada um 
deles, que possuem uma relação de conflito e congruência entre si. Convém salientar que a estrutura de valores apresenta compatibilidades e contradições entre os domínios, ou seja, de acordo com o modelo é contraditório dar alta prioridade a dois domínios conceitualmente distantes ao mesmo tempo (SCHWARTZ e BILSKY, 1987). Dessa forma, os tipos motivacionais de valores encontram-se organizados em duas dimensões bipolares: abertura à mudança versus conservação, e autopromoção versus auto-transcendência (SCHWARTZ, 1994). A figura 1 apresenta os tipos motivacionais relacionados aos objetivos que esses expressam as duas dimensões bipolares.

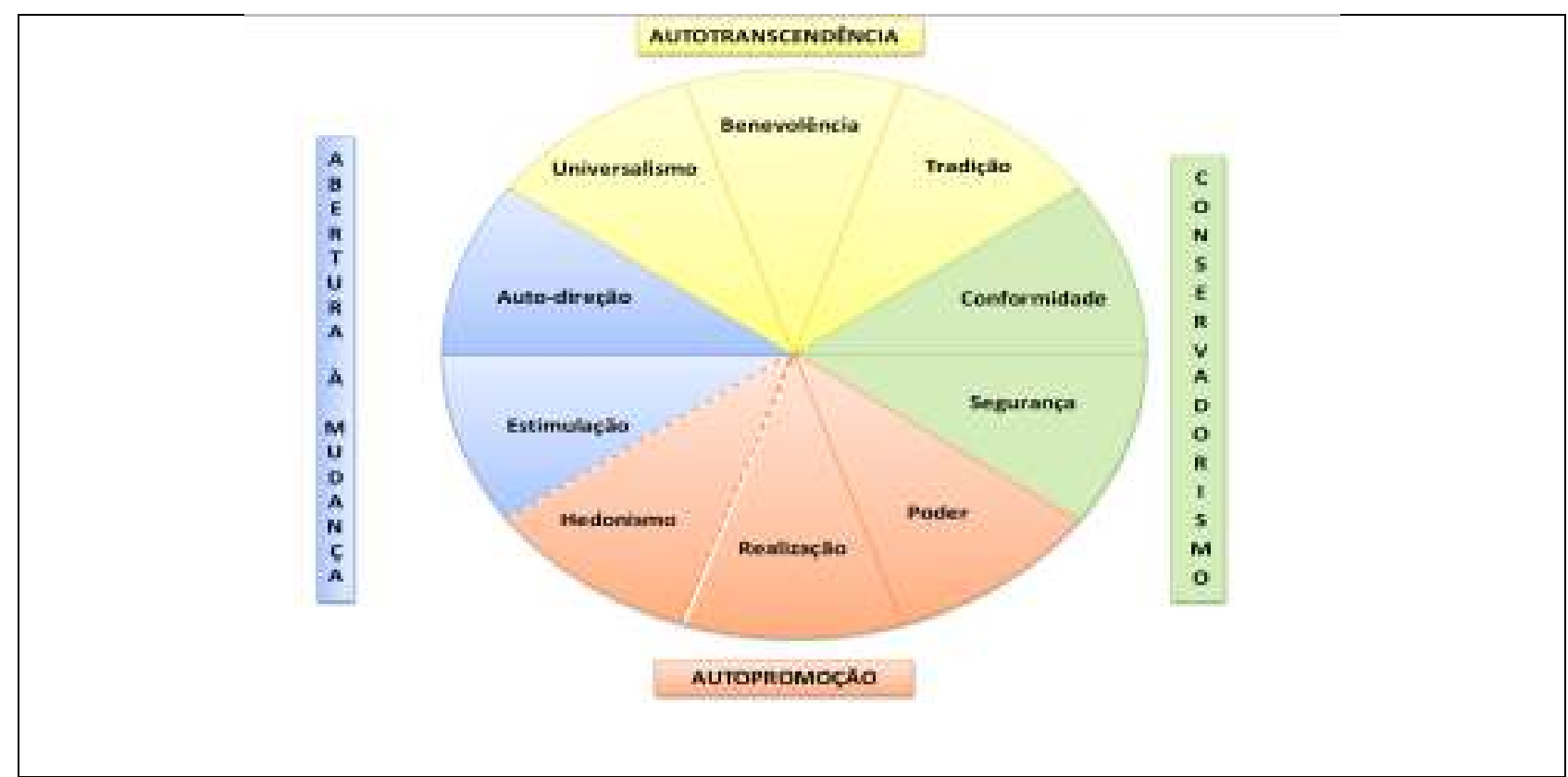

Figura 1: Estrutura Motivacional dos Valores Humanos

Fonte: Adaptado de Schwartz (1992, 1994).

A primeira dimensão reflete o conflito entre a busca por mudança e o conservadorismo, isto é, entre a ênfase no pensamento e nas ações, independentes do indivíduo, à mudança e a manutenção da ordem e respeito à tradição (SCHWARTZ, 1994). De acordo com Schwartz (1994), em um extremo dessa dimensão encontram-se os valores ligados à inovação, criação, autonomia e abertura aos desafios, e, em outro extremo, os valores relacionam-se a busca de estabilidade, segurança, ordem social, autocontrole e respeito à tradição.

A segunda dimensão trata do paradoxo existente entre autopromoção e autotranscendência, isto é entre a ênfase na aceitação dos outros como iguais e a preocupação com o seu bem-estar, a busca pelo próprio sucesso relativo e domínio sobre os outros (SCHWARTZ, 1994). Segundo Schwartz (1994), em um extremo dessa dimensão, encontram-se os valores ligados ao controle sobre as pessoas, prestígio, prazer e gratificação para si e em outro extremo a tolerância, compreensão e promoção do bem-estar de todos e da natureza. 


\section{VALORES RELATIVOS AO TRABALHO: MOTIVOS QUE LEVAM A TRABALHAR}

De acordo com Porto e Tamayo (2003), os indivíduos possuem uma estrutura geral de valores, relativa aos seus valores pessoais. Acrescenta-se a essa discussão o entendimento acerca de valores relativos a determinados contextos específicos da vida do indivíduo. Nesse sentido, partilha-se do entendimento de Roe e Ester (1999), ao enfatizarem a importância dos valores relativos ao trabalho, devido ao papel que o trabalho representa na vida social, não somente como forma de sustento, mas também por ser considerado, dentre outros aspectos, como base para participação e status social.

No entender de Lyons, Duxbury e Higgins (2006), os valores relativos ao trabalho são definidos como a opinião generalizada sobre a conveniência de certos atributos do trabalho (remuneração, autonomia, condições de trabalho), e os resultados relacionados ao trabalho (realização e prestígio). Da mesma forma que os valores gerais, os valores relativos ao trabalho, atuam como critérios que um indivíduo utiliza na escolha apropriada de comportamentos e metas relacionadas ao trabalho, ou seja, respondem à questão acerca do que é importante para os indivíduos em sua vida no trabalho (LYONS, HIGGINS e DUXBURY, 2010).

Assim, nesse cenário, os valores são entendidos como os princípios ou crenças sobre metas ou recompensas desejáveis, hierarquicamente organizados, que os indivíduos buscam por meio do trabalho e que guiam as suas avaliações sobre os resultados e o contexto do trabalho (PORTO e TAMAYO, 2007). Nessa mesma linha, Ros, Schwartz e Surkiss (1999) definem valores de trabalho como sendo crenças sobre estados-fim, ou comportamentos desejáveis, em que as diferentes metas são ordenadas por sua importância, como guiando princípios para avaliar ambientes e resultados do trabalho, bem como para escolher entre alternativas diferentes de trabalho.

Diante da definição proposta por Porto e Tamayo (2007), pode-se perceber a existência de três aspectos conexos aos valores relativos ao trabalho: (i) cognitivo, relacionado às crenças sobre o que é o não desejável no trabalho; (ii) motivacional, relacionado aos interesses e desejos do indivíduo em relação ao trabalho; (iii) hierárquico, no qual os valores são hierarquizados e classificados em um continuum de importância.

Convém acrescentar a essa discussão esforços teóricos e empíricos no sentido de estabelecer tipologias e formas de mensuração dos valores relativos ao trabalho. Em âmbito nacional, um dos modelos de notável relevância no estudo dos valores relativos diz respeito à a Escala de Valores Relativos ao Trabalho (EVT), desenvolvida por Porto e Tamayo (2003).

Essa escala, instrumentaliza os valores como príncipios orientadores da vida do indivíduo no trabalho. A EVT é constituída por quatro categorias: (i) realização no trabalho, que se refere à busca do prazer e realização pessoal e profissional, bem como de independência de pensamento e ação no trabalho por meio da autonomia intelectual e criatividade; (ii) relações sociais, referente a busca por parte do indivíduo em manter relações sociais positivas no trabalho e de contribuição para a sociedade por meio do trabalho; (iii) prestígio, como a busca de autoridade, sucesso profissional e poder de influência no trabalho; e, (iv) estabilidade, referente à busca de segurança e ordem na vida por meio do trabalho, possibilitando suprir materialmente as necessidades pessoais. 
Tendo em vista que a Escala de Valores relativos ao Trabalho (EVT) foi desenvolvida tendo como base os estudos de Schwartz (1992, 1994), suas dimensões apresentam interface com a estrutura de valores básicos proposta por esse autor, inclusive no que tange à relação de conflito e congruência entre dimensões opostas (PORTO e TAMAYO, 2007). Assim, observa-se que as dimensões da EVT possuem relação direta com as dimensões propostas por Schwartz $(1992,1994)$, como pode ser visualizado no Quadro 1.

Quadro 1 - Valores Básicos e Valores Relativos ao Trabalho

\begin{tabular}{|c|c|c|c|}
\hline Dimensão & Tipos & Metas & $\begin{array}{l}\text { Relação com a } \\
\text { EVT }\end{array}$ \\
\hline \multirow{3}{*}{ Abertura a Mudança } & Autodeterminação & Independência de pensamento, ação e opção. & \multirow{3}{*}{$\begin{array}{l}\text { Realização no } \\
\text { trabalho }\end{array}$} \\
\hline & Estimulação & Excitação, novidade, mudança e desafio. & \\
\hline & Hedonismo & Prazer e gratificação sensual para si mesmo. & \\
\hline \multirow{2}{*}{ Autopromoção } & Realização & $\begin{array}{l}\text { Sucesso pessoal obtido por meio de uma } \\
\text { demonstração de competência. }\end{array}$ & \multirow{2}{*}{ Prestígio } \\
\hline & Poder Social & Controle sobre pessoas e recursos, prestígio. & \\
\hline \multirow{3}{*}{ Conservação } & Segurança & $\begin{array}{l}\text { Integridade social, estabilidade da sociedade, } \\
\text { estabilidade do relacionamento e de si mesmo. }\end{array}$ & \multirow{3}{*}{ Estabilidade } \\
\hline & Conformidade & $\begin{array}{l}\text { Controle de impulsos e ações que podem violar } \\
\text { normas sociais ou prejudicar aos outros. }\end{array}$ & \\
\hline & Tradição & $\begin{array}{l}\text { Respeito e aceitação dos ideais e costumes da } \\
\text { sociedade. }\end{array}$ & \\
\hline \multirow{2}{*}{ Autotranscendência } & Benevolência & Promoção do bem-estar das pessoas íntimas. & \multirow{2}{*}{ Relações sociais } \\
\hline & Universalismo & $\begin{array}{l}\text { Tolerância, compreensão e promoção do bem-estar } \\
\text { de todos e da natureza. }\end{array}$ & \\
\hline
\end{tabular}

Fonte: Elaborado com base em Schwartz (1992, 1994), Porto e Tamayo $(2003,2007)$

Assim, o fator realização no trabalho está associado positivamente à abertura à mudança, pelo fato de favorecer a mudança através do trabalho autônomo e criativo; as relações sociais se associam positivamente a autotranscendência, uma vez que o trabalho proporciona o alcance de relacionamento social positivo; o fator prestígio possui uma relação com a autopromoção por proporcionar o alcance das metas pessoal e influências sobre os outros; por fim o fator estabilidade está associado à conservação, ou seja, a segurança e manutenção do status quo por meio do trabalho (PORTO e TAMAYO, 2003, 2007). 


\section{MÉTODO}

Visando atender aos objetivos delineados neste estudo, realizou-se uma pesquisa descritiva, de caráter quantitativo tendo como objeto de estudo uma Instituição Federal de Ensino Superior, localizada no interior do estado do Rio Grande do Sul.

Os critérios para a escolha da organização estudada devem-se aos seguintes fatores: (i) ser uma das primeiras Instituições de Ensino Superior a se instalar no interior do Brasil; (ii) pelo crescimento que vem despontando no cenário nacional, devido ao processo de expansão universitária que está ocorrendo, por meio da ampliação do número de vagas, criação de novos cursos, implantação de cursos à distância e instituição de novos pólos de Ensino em outras regiões do Estado do Rio Grande do Sul; e (iii) pela importância que representa para região onde está situada com relação ao papel social que desempenha.

A instituição possui cerca de 3800 funcionários, sendo desses 2642 servidores técnicos administrativos em educação. Para fins deste estudo, optou-se por pesquisar os servidores técnicos administrativos em educação, vinculados a administração central da instituição (reitoria), por entender que esses participam do desenvolvimento de atividades de planejamento e estratégias para a instituição como um todo. Nesse caso, partilha-se do entendimento de Tamayo e Trócolli (2002), que enfatizam que as organizações atuam através do comportamento individual dos seus membros-chave, ou seja, daqueles que ocupam posições gerenciais. Atualmente, existem cerca de 590 funcionários trabalhando na administração central, sendo que desses, 240 integraram o presente estudo. $O$ tipo de amostragem foi não probabilística por conveniência, uma vez que os indivíduos eram convidados a participar do estudo, momento no qual, o pesquisador fornecia informação sobre os objetivos do estudo, esclarecimentos referentes ao anonimato e ao sigilo. Foi garantido o caráter voluntário da participação, a possibilidade de desistência a qualquer momento da pesquisa, bem como o respeito aos preceitos éticos que regem a realização de pesquisas com seres humanos.

A situação escolhida para a coleta de dados foi a aplicação de um questionário de forma individual, realizada no próprio ambiente de trabalho. Esse questionário foi composto por duas partes: a primeira foi composta por questões que buscavam traçar o perfil dos respondentes, identificando dados pessoais como: sexo, faixa etária, estado civil, escolaridade, nível de formação e dados organizacionais como: cargo, tempo de instituição e tempo no cargo. A segunda parte foi estruturada a partir da Escala de Valores Relativos ao Trabalho, desenvolvida e validada por Porto e Tamayo (2003). Essa escala consta de 45 itens, agrupados em quatro fatores: Relações Sociais, Realização Profissional, Prestígio e Estabilidade.

Para a medição do nível de concordância das afirmativas com relação à escala de valores relativos ao trabalho, foi utilizada uma escala do tipo Likert, que contou com cinco pontos, onde a atribuição do número 1 representa "Nada Importante" e o número 5, "Extremamente Importante". Assim, convém salientar que quanto mais próximo de " 5 " maior o grau de importância atribuída ao fator, enquanto que, quanto mais próximo de "1" menor o grau de importância.

O questionário foi submetido à validação semântica e de conteúdo através de uma avaliação por três especialistas, pesquisadores da área. Esses avaliaram o conteúdo e a forma dos questionários quanto a sua clareza, objetividade e precisão de linguagem. Feito isso, realizou-se um pré-teste, com 08 indivíduos com o objetivo de foi verificar as possíveis inconsistências (complexidade das questões, imprecisão na redação, 
questões desnecessárias, constrangimentos ao informante, exaustão, dentre outras) que o instrumento de coleta de dados poderia apresentar e, assim, identificar possíveis melhorias no mesmo. É importante destacar que os dados do pré-teste não foram considerados na amostra.

A consistência interna da segunda parte do questionário foi medida por meio do alpha de cronbach. Na presente pesquisa, obteve-se um alpha de Cronbach muito próximo ao encontrado no estudo original de Porto e Tamayo (2003). Todos os índices obtidos foram superiores a 0,6 confirmando a confiabilidade interna do instrumento (MALHOTRA, 2006). No que se refere à confiabilidade do instrumento de pesquisa desenvolvido pelos referidos autores, em seu estudo original foram obtidos os seguintes coeficientes Alpha de Cronbach: 0,88 para o fator Realização no Trabalho; 0,88 para o fator Relações Sociais; 0,87 para o fator Prestígio; e 0,81 para o fator Estabilidade, contra 0,863 no fator Realização no trabalho; 0,856 no fator Relações sociais; 0,863 no fator Sociais Prestígio; 0,798 no fator Estabilidade obtidos no presente estudo o que garante a confiabilidade do instrumento de pesquisa utilizado. Para elucidar a forma com que serão atingidos os objetivos do estudo, bem como as categorias analíticas utilizadas, foi elaborado o Quadro 2, abaixo.

Quadro 2 - Objetivos do Estudo, Categorias, Fatores e Técnicas de Análise.

\begin{tabular}{|c|c|c|c|}
\hline Objetivos do Estudo & Categorias & Fatores & Análise dos Dados \\
\hline $\begin{array}{l}\text { Identificar a estrutura hierárquica } \\
\text { de valores relativos ao trabalho }\end{array}$ & $\begin{array}{l}\text { Hierarquia de } \\
\text { Valores Relativos } \\
\text { ao trabalho } \\
\text { (EVT - Porto e } \\
\text { Tamayo, 2003) }\end{array}$ & $\begin{array}{l}\text { Estabilidade, Realização } \\
\text { no Trabalho, Relações } \\
\text { Sociais e Prestígio. }\end{array}$ & $\begin{array}{l}\text { Análise fatorial, médias, } \\
\text { desvio-padrão, teste t para } \\
\text { amostras pareadas e } \\
\text { coeficiente de correlação de } \\
\text { Pearson. }\end{array}$ \\
\hline \multirow{2}{*}{$\begin{array}{l}\text { Identificar as diferenças de } \\
\text { percepção em relação às variáveis } \\
\text { pessoais e organizacionais }\end{array}$} & $\begin{array}{l}\text { Variáveis } \\
\text { Pessoais }\end{array}$ & $\begin{array}{l}\text { Gênero, Idade, estado } \\
\text { civil, grau de instrução. }\end{array}$ & \multirow{2}{*}{$\begin{array}{l}\text { Analise descritiva da } \\
\text { amostra, teste F para } \\
\text { análise de variâncias, teste } t \\
\text { para amostras } \\
\text { independentes. }\end{array}$} \\
\hline & $\begin{array}{l}\text { Variáveis } \\
\text { Organizacionais }\end{array}$ & $\begin{array}{l}\text { Tempo de cargo, tempo } \\
\text { de serviço, número de } \\
\text { setores nos quais } \\
\text { trabalhou. }\end{array}$ & \\
\hline
\end{tabular}

Fonte: Elaborado pelos autores

Para a análise dos dados, utilizou-se o software "Statistical Package for the Social Sciences - SPSS 13.0", a fim de realizar os testes estatísticos descritivos (de tendência central e de dispersão), indicador de consistência interna Alpha de Crombach, análise fatorial (principal axisfactoring), rotação ortogonal varimax com normalização (Kaiser-Meyer-Olkine (KMO) e teste Esfericidade de Bartlett), os quais confirmaram a adequação dos dados para o estudo fatorial. Para o relacionamento entre as variáveis realizou-se o coeficiente de correlação de Pearson e teste t para diferenças de médias, detalhados na seção de resultados.

\section{RESULTADOS}

Com relação ao perfil dos pesquisados, 132 são do sexo masculino (55\%) e 108 são do sexo feminino (45\%). Sobre a escolaridade dos mesmos, percebe-se que a maioria possui Pós-Graduação (38,8\%). O cargo de maior representatividade foi o de nível médio (50,8\%), seguido do de nível superior (33,3\%). É importante ressaltar que 72 funcionários (30\%) exercem cargo de chefia. Quanto à idade dos pesquisados, obteve-se 
uma média de 45,12 anos. Já com relação ao estado civil dos respondentes, predominaram pessoas casadas (52,1\%). Com relação ao tempo de Instituição dos funcionários, obteve-se uma média de 18 anos, sendo que esses já trabalharam em média de 1,88 setores. Quanto ao tempo no cargo que os funcionários ocupam atualmente, a média foi 11,94 anos.

A análise fatorial realizada neste estudo gerou quatro fatores, confirmando as dimensões encontradas por Porto e Tamayo (2003). No que tange à composição dos fatores, pode-se perceber que se manteve a essência daqueles obtidos no estudo original, uma vez que apenas para o segundo fator obtiveram-se alterações nas variáveis, sendo que este fator resultou em 13 questões, 2 correspondentes ao fator 1 e uma ao fator 3

Para fins de análise, foram calculados as médias e os desvios-padrão para cada variável, bem como para os fatores, os quais, juntamente com os resultados dos coeficientes Alpha de Cronbach, encontram-se dispostos no Quadro 3.

A análise dos dados evidenciou que os pesquisados foram, de um modo geral, coerentes entre si, visto que foram obtidos valores de desvio-padrão inferiores a um para a maioria dos fatores, conforme indicado por HAIR et al (2009). Contudo, cabe salientar que, em relação ao fator Prestígio, houve uma maior divergência entre as respostas dos sujeitos pesquisados, uma vez que a maioria dos resultados obtidos para o desvio-padrão das variáveis que compõem este fator foram superiores a um.

No que se refere à composição final dos fatores, pode-se perceber que apenas o fator 2 incluiu questões provenientes de outros fatores. Este fator abarcou duas questões relativas ao fator 1 (trabalho variado e enfrentar desafios), relacionadas à natureza do trabalho, e uma questão relativa ao fator 3 (identificar-me com o trabalho), relacionada ao próprio indivíduo.

Quadro 3 - Valores Relativos ao Trabalho

continua

\begin{tabular}{|c|c|c|c|c|}
\hline Fatores & Variáveis & Fator Original & Média & Desvio \\
\hline \multirow{7}{*}{$\begin{array}{l}\text { Fator } 1 \text { - } \\
\text { Realização no } \\
\text { Trabalho }\end{array}$} & 10. Ter prazer no que faço & Fator 1 & 4,3235 & 0,8115 \\
\hline & 13. Trabalho interessante & Fator 1 & 3,9580 & 0,7891 \\
\hline & 39. Ser feliz com o trabalho que realizo & Fator 1 & 4,2479 & 0,7912 \\
\hline & 16. Gostar do que faço & Fator 1 & 4,2059 & 0,9115 \\
\hline & 7. Trabalho intelectualmente estimulante & Fator 1 & 3,7899 & 0,9711 \\
\hline & 30. Realização pessoal & Fator 1 & 4,0630 & 0,9369 \\
\hline & 5. Realizar um trabalho significativo para mim & Fator 1 & 4,1429 & 0,8743 \\
\hline \multirow{6}{*}{$\alpha=0,897$} & 12. Satisfação pessoal & Fator 1 & 4,2479 & 0,7965 \\
\hline & 14. Crescimento intelectual & Fator 1 & 4,0504 & 0,9309 \\
\hline & 44. Trabalho que requer originalidade e criatividade & Fator 1 & 3,6597 & 0,9127 \\
\hline & 4. Realização profissional & Fator 1 & 4,3193 & 0,7399 \\
\hline & 41. Aprimorar conhecimentos da minha profissão & Fator 1 & 4,0294 & 0,9066 \\
\hline & $\begin{array}{l}\text { 8. Autonomia para estabelecer a forma de realização do } \\
\text { trabalho }\end{array}$ & Fator 1 & 3,9622 & 0,8284 \\
\hline \multicolumn{3}{|c|}{ Total do Fator } & 4,0769 & 0,8616 \\
\hline
\end{tabular}


Quadro 3 - Valores Relativos ao Trabalho

continuação

\begin{tabular}{|c|c|c|c|c|}
\hline Fatores & Variáveis & Fator Original & Média & Desvio \\
\hline \multirow{12}{*}{$\begin{array}{l}\text { Fator } 2 \text { - Relações } \\
\text { Sociais }\end{array}$} & 36. Ajudar os outros & Fator 2 & 3,9367 & 0,9021 \\
\hline & 20. Auxiliar os colegas de trabalho & Fator 2 & 4,0802 & 0,8424 \\
\hline & 29. Colaborar para o desenvolvimento da sociedade & Fator 2 & 3,7553 & 0,9247 \\
\hline & 3. Combater injustiças sociais & Fator 2 & 3,7131 & 0,9753 \\
\hline & 19. Ser útil para a sociedade & Fator 2 & 3,9831 & 0,8876 \\
\hline & 28. Ter compromisso social & Fator 2 & 3,6582 & 0,9418 \\
\hline & $\begin{array}{l}\text { 45. Colaborar com colegas de trabalho para alcançar as } \\
\text { metas de trabalho do grupo. }\end{array}$ & Fator 2 & 4,1266 & 0,8690 \\
\hline & 23. Bom relacionamento com colegas de trabalho & Fator 2 & 4,2911 & 0,7890 \\
\hline & 26. Amizade com colegas de trabalho & Fator 2 & 3,9494 & 0,8958 \\
\hline & 40. Trabalho variado & Fator 1 & 3,4958 & 0,9221 \\
\hline & 38. Enfrentar desafios & Fator 3 & 3,8017 & 0,8870 \\
\hline & 24. Identificar-me com o trabalho & Fator 1 & 3,9832 & 0,8857 \\
\hline \multicolumn{3}{|c|}{ Total do Fator } & 3,9431 & 0,8917 \\
\hline \multirow{10}{*}{ Fator 3 - Prestígio } & 42. Obter posição de destaque & Fator 3 & 2,3713 & 1,0995 \\
\hline & 25. Supervisionar outras pessoas & Fator 3 & 2,5612 & 1,1015 \\
\hline & 33. Ter fama & Fator 3 & 1,8523 & 1,0081 \\
\hline & $\begin{array}{l}\text { 27. Competir com colegas de trabalho para alcançar as } \\
\text { minhas metas profissionais }\end{array}$ & Fator 3 & 2,1519 & 1,1322 \\
\hline & 22. Ter prestígio & Fator 3 & 2,9958 & 1,0951 \\
\hline & 17. Status no trabalho & Fator 3 & 2,7300 & 1,1400 \\
\hline & 31. Ter superioridade baseada no êxito do meu trabalho & Fator 3 & 2,7426 & 1,1740 \\
\hline & 34. Ter notoriedade & Fator 3 & 2,2911 & 1,1948 \\
\hline & 15. Seguir a profissão da família & Fator 3 & 1,9578 & 1,1637 \\
\hline & 6. Competitividade & Fator 3 & 2,7848 & 1,0537 \\
\hline \multicolumn{3}{|c|}{ Total do Fator } & 2,4663 & 1,1169 \\
\hline \multirow{7}{*}{$\begin{array}{l}\text { Fator } 4- \\
\text { Estabilidade }\end{array}$} & 1. Estabilidade financeira & Fator 4 & 4,1055 & 0,8934 \\
\hline & 43. Ter melhores condições de vida & Fator 4 & 4,0506 & 0,8767 \\
\hline & 9. Poder me sustentar & Fator 4 & 4,3460 & 0,8724 \\
\hline & 2. Ser independente financeiramente & Fator 4 & 4,2827 & 0,8735 \\
\hline & 18. Ganhar dinheiro & Fator 4 & 3,6118 & 0,9661 \\
\hline & 35. Estabilidade no trabalho & Fator 4 & 4,1139 & 0,9111 \\
\hline & 37. Suprir necessidades materiais & Fator 4 & 3,6076 & 0,8349 \\
\hline \multicolumn{3}{|c|}{ Total do Fator } & 4,1055 & 0,8767 \\
\hline
\end{tabular}

Fonte: Elaborado pelos autores com base nos dados da pesquisa. 
Por meio da análise dos dados constantes no Quadro 4, pode-se observar que a maior média obtida foi para o fator Estabilidade (média $=4,1055$ ). Ao analisar esse fator, observa-se que a questão que obteve maior média (média $=4,3460$ ) foi a questão (9) "Poder me sustentar". Nesse sentido, pode-se observar que os funcionários valorizam aspectos relacionados à remuneração no que diz respeito à estabilidade financeira.

Tendo em vista que, de acordo com Porto e Tamayo (2003), os valores relativos ao trabalho demonstram aquilo que é importante, desejável e preferível para o indivíduo no contexto do trabalho, a maior valorização do fator Estabilidade sinaliza que os sujeitos pesquisados atribuem maior importância à busca de segurança e ordem na vida, através do trabalho, possibilitando suprir suas necessidades materiais.

Por outro lado, o fator que obteve a menor média foi o fator Prestígio (média $=2.4663$ ). No que se refere a esse fator, pode-se observar que a menor média foi para a questão (33) "Ter fama" (1,8523). Assim, a menor valorização para o fator prestígio pode revelar que os funcionários da instituição pesquisada não estão focados na busca por autoridade, sucesso profissional e poder de influência no trabalho (PORTO, 2005). Ainda, os resultados obtidos para este fator se assemelham aos obtidos por Goés (2006), que, ao analisar os valores relativos ao trabalho em uma instituição financeira pública, obteve médias inferiores para o fator prestígio.

Considerando que as médias obtidas resultaram em valores muito próximos, realizou-se o teste t para amostras emparelhadas visando verificar se as médias encontradas são estatisticamente significativas. Esse teste foi escolhido, pois o conjunto de dados analisado relaciona-se ao mesmo grupo de respondentes (MALHOTRA, 2006). A Tabela 1 ilustra o teste t para os valores relativos ao trabalho.

Tabela 1 - Teste t Para os Valores Relativos ao Trabalho

\begin{tabular}{|c|c|c|c|c|c|}
\hline \multirow{2}{*}{ Pares } & \multicolumn{2}{|c|}{ Diferenças entre os Pares } & \multirow{2}{*}{$\begin{array}{c}\text { Estatística } \\
\mathrm{t}\end{array}$} & \multirow{2}{*}{$\begin{array}{l}\text { Graus de } \\
\text { liberdade }\end{array}$} & \multirow{2}{*}{ Significância } \\
\hline & Média & Desvio-Padrão & & & \\
\hline Realização Trabalho - Relações Sociais & 0,32598 & 0,43804 & 11,481 & 237 & 0,000 \\
\hline Realização Trabalho - Prestigio & 1,62755 & 0,83640 & 30,020 & 237 & 0,000 \\
\hline Realização Trabalho - Estabilidade & 0,06152 & 0,69471 & 1,366 & 237 & 0,173 \\
\hline Prestigio - Estabilidade & $-1,56604$ & 0,89197 & $-27,086$ & 237 & 0,000 \\
\hline Relações Sociais - Prestigio & 1,30157 & 0,74082 & 27,105 & 237 & 0,000 \\
\hline Relações Sociais - Estabilidade & $-0,26446$ & 0,67379 & $-6,055$ & 237 & 0,000 \\
\hline
\end{tabular}

Nota: valores significativos se $p<0,050$

Fonte: Dados da pesquisa

A partir dos dados apresentados na Tabela 1, observa-se que as diferenças entre as médias dos fatores da EVT são estatisticamente significativas, exceto entre os fatores Realização no Trabalho e Estabilidade, uma vez que o valor de Sig encontrado é maior que 0,05 . Esse resultado pode sinalizar que os pesquisados apresentam percepções semelhantes em relação a esses fatores, ou seja, tanto Estabilidade quanto Realização no Trabalho podem ter igual importância na percepção acerca dos motivos que levam a trabalhar. 
Os resultados remetem à compreensão de que os pesquisados buscam alcançar, por meio do trabalho, segurança e ordem na vida, a fim de suprir materialmente suas necessidades pessoais (PORTO, 2005), mas, concomitantemente a isso, buscam realização no trabalho, desejando crescimento pessoal, autonomia, interesse e realização de atividades intelectualmente estimulantes e desafiadoras (ROS, SCHWARTZ e SURKISS, 1999; PORTO TAMAYO, 2003, 2007). Os resultados encontrados neste estudo sinalizam para o fato de que, na percepção dos funcionários da instituição pesquisada, os valores relativos ao trabalho apresentam a seguinte hierarquia: Estabilidade, Realização no Trabalho, Relações Sociais e Prestígio.

No intuito de compreender como os valores relativos ao trabalho se relacionam entre si, realizou-se o cálculo do coeficiente de correlação de Pearson, o qual visa fornecer a associação linear entre as variáveis (AAKER, KUMAR e DAY, 2001), disposto na Tabela 2.

Tabela 2 - Correlação entre os Valores Relativos ao Trabalho

\begin{tabular}{lcccc}
\hline & Realização & Relações Sociais & Prestígio Estabilidade \\
\hline Realização & 1,000 & & & \\
Relações Sociais & $0,706^{* *}$ & 1,000 & & \\
Prestígio & $0,309^{* *}$ & $0,357^{* *}$ & 1,000 & \\
Estabilidade & $0,305^{* *}$ & $0,320^{* *}$ & $0,161^{* *}$ & 1,000 \\
\hline
\end{tabular}

** Correlação é significativa ao nível de 0,01

Fonte: Dados da pesquisa

No que tange às relações entre os fatores, pode-se observar, conforme os dados constantes na Tabela 2, que todas as correlações encontradas são significativas e diretas, o que significa que todos os fatores da EVT, no caso deste estudo, variam no mesmo sentido (PESTANA e GAGEIRO, 2003).

Um fato que merece destaque é que, de acordo com Porto e Tamayo (2003), os fatores Realização no Trabalho e Estabilidade, assim como os fatores Prestígio e Realização, representam dimensões opostas, devido à interface entre tais fatores e a teoria de Schwartz $(1992,1994)$, evidenciando uma relação antagônica entre os mesmos. Esse fato não foi evidenciado neste estudo, uma vez que não foram obtidas correlações negativas entre tais fatores, demonstrando não existir, para a amostra estudada, a relação de oposição entre os tipos motivacionais evidenciada na literatura.

No tocante à intensidade das correlações encontradas, os dados da pesquisa mostram que a maioria das correlações possui intensidade baixa, devido estarem no intervalo compreendido entre 0,2 e 0,39 (PESTANA e GAGEIRO, 2003), com exceção da correlação entre as variáveis Relações Sociais e Realização no Trabalho, a qual possui intensidade alta $(0,706)$; e entre as variáveis Prestígio e Estabilidade, que possui intensidade de associação muito baixa $(0,161)$ (PESTANA e GAGEIRO, 2003).

O maior valor encontrado para as correlações $(0,706)$ ocorreu entre os fatores Realização no Trabalho e Relações Sociais. Dessa forma, nota-se que, na percepção dos funcionários pesquisados, a busca por prazer, realização pessoal e independência de pensamento e ação no trabalho está altamente correlacionada com a busca por relações sociais positivas por meio do trabalho. Por outro lado, a menor correlação $(0,161)$ ocorreu entre as variáveis Estabilidade e Prestígio. Esse resultado evidencia que, na percepção dos sujeitos 
pesquisados, há uma baixa associação entre a busca por suprir as necessidades materiais através do trabalho e o sucesso profissional e o poder de influência no mesmo.

Visando ampliar a compreensão acerca dos motivos que levam indivíduos com diferentes características a trabalhar e a permanecer em uma instituição de ensino superior pública, procedeu-se a realização do teste t para amostras independentes entre as variáveis de perfil e organizacionais e os fatores relacionados à estrutura da EVT obtida. Dentre as variáveis testadas, foram obtidas diferenças significativas de médias para as variáveis: gênero, idade, tempo de serviço, tempo de cargo, número de setores atuação em cargos de chefia.

No que tange ao gênero, os resultados do teste $t$ foram significativos para os fatores realização no trabalho $(t=0,242, p<0,05)$, para o qual o teste $t$ foi homocedástico; e para o fator estabilidade $(t=2,228, p$ $<0,05)$, para o qual o teste $t$ foi heterocedástico. Para ambos os fatores, considerando um nível de significância de $5 \%$, as médias atribuídas pelas mulheres foram superiores às médias atribuídas pelos homens (Realização

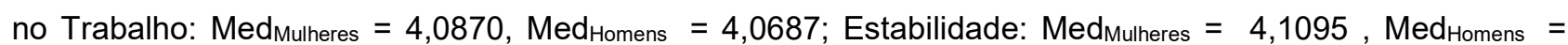
3,9386).

Tais resultados indicam que as mulheres tendem a uma maior valorização de realização e estabilidade, no trabalho. Tais achados vão ao encontro do postulado por Silva, Mendonça e Zanini (2010), que ao realizar um estudo sobre a diferença do gênero e os valores relativos ao trabalho, obtiveram resultados que indicam maior valorização dos referidos fatores por parte das mulheres.

Dada a importância da compreensão das diferenças entre os gêneros, principalmente no contexto do trabalho, torna-se necessário ampliar o entendimento acerca dos fatores para os quais as diferenças entre homens e mulheres se mostraram significativas. Assim, serão analisadas as variáveis que compõe os fatores Realização no Trabalho e Estabilidade.

Analisando as variáveis que compõe o fator Realização no Trabalho, observam-se médias significativas ( $t=0,164 ; p<0,05)$ para: "(08) Autonomia para a estabelecer a forma de realização do trabalho", no qual as médias das mulheres $(3,9720)$ são superiores em relação às medias atribuídas pelos homens $(3,9542)$. Além disso, as médias desta dimensão mostraram-se significativas $(t=0,159 ; p<0,05)$ para a variável "(44) Trabalho que requer originalidade e criatividade", para o qual as médias das mulheres $(3,6636)$ são superiores às atribuídas pelos homens $(3,6565)$.

A análise das médias das variáveis que compõe o fator Estabilidade demonstra diferenças significativas para três, das sete variáveis avaliadas, sendo que, em todos os casos, as médias atribuídas pelas mulheres foram estatisticamente superiores às atribuídas pelos homens. Assim, os resultados indicam que, em relação à busca por estabilidade através do trabalho, as mulheres atribuem maior valorização aos aspectos relacionados à estabilidade financeira (variável $1 ; t=2,242, p<0,05$ ), independência financeira (variável 2; $t=2,235, p<0,05$;) e suprimento das necessidades materiais (variável $37 ; t=3,186, p<0,05$ ). Nesse sentido, Silva, Mendonça e Zanini (2010) afirmam que a tendência feminina em priorizar a estabilidade pode estar relacionada ao crescente papel da mulher como provedora familiar, assumindo responsabilidades relacionadas à manutenção da família.

Para a variável idade, foi calculada a idade média dos pesquisados, obtendo-se um valor de idade média de 45 anos. A realização do teste $t$, para essa variável sinalizou diferenças estatisticamente significantes para os fatores Prestígio $(t=2,488 ; p<0,05)$ e Relações Sociais $(t=2,661 ; p<0,05)$, sendo 
realizado teste t homocedástico e heterocedástico, respectivamente. Em ambos os casos, observou-se que os funcionários com idade superior a idade média atribuíram maior importância aos referidos fatores do que aqueles com idade inferior a idade média, isto é, (Prestígio: $M_{e d}>45$ anos $=2,5360, M_{<}<45$ anos $=2,2891$; Relações Sociais: Med>=45 anos $=3,8276, M_{<}<45$ anos $\left.=3,6304\right)$.

Assim, os resultados demonstram que os funcionários com idade superior à idade média buscam sucesso profissional e poder de influência no trabalho, concomitantemente com a manutenção de relações sociais positivas no trabalho (PORTO e TAMAYO, 2003). Em relação ao fator prestígio, os resultados vão ao encontro dos achados de Jing e Rounds (2011). Os autores analisam estabilidade e mudanças em relação aos valores relativos ao trabalho, obtendo resultados que indicam que, com o tempo, os indivíduos tendem a valorizar a obtenção de status e prestígio através do trabalho (JING e ROUNDS, 2011).

No que concerne às diferenças de percepções em relação às variáveis organizacionais (isto é, vinculadas às características da relação indivíduo-organização), os resultados sinalizaram diferenças significativas de médias para as variáveis: tempo de serviço, tempo no cargo e posição na organização. Dessas, apenas para a variável tempo de cargo levou a realização do teste t homocedástico. Dado a igualdade de variâncias, nas demais variáveis, o teste realizado foi do tipo heterocedástico.

Quanto ao tempo de serviço, o teste $t$ foi realizado tendo como ponto de corte entre os grupos o tempo médio de serviço dos pesquisados (18 anos). Assim, os resultados do teste $t$ demonstram diferenças significativas de médias para o fator Realização no Trabalho $(t=-1,098, p<0,05)$, para o qual os pesquisados com tempo de serviço igual ou superior ao tempo médio atribuíram médias superiores $(3,7870)$ quando comparados às médias atribuídas por aqueles com tempo de serviço inferior $(3,7411)$. Esses resultados demonstram que, os pesquisados que possuem maior tempo de serviço na organização buscam, através do trabalho, realização pessoal e profissional (PORTO e TAMAYO, 2003).

No que concerne ao tempo de cargo dos pesquisados, o teste foi realizado tendo como base o tempo médio de cargo dos pesquisados (12 anos). O resultado do teste t sinalizou para diferenças significativas de médias para o fator Relações Sociais $(t=-1,096, p<0,05)$, sendo que as médias atribuídas por profissionais que estão no cargo por um tempo igual ou superior ao termo médio são estatisticamente superiores $(4,1201)$, em relação às médias dos pesquisados com tempo de cargo inferior ao tempo médio $(4,0350)$.

No que concerne às diferenças de percepção em relação à posição que o respondente ocupa na organização estudada, analisaram-se as diferenças de percepção entre aqueles que ocupam cargos de chefia ou não, uma vez que tais cargos são considerados estratégicos, e determinam as diretrizes e políticas da instituição. Nesse sentido, os resultados obtidos demonstram haver diferenças significativas para apenas para o fator Relações Sociais ( $t=3,183, p<0,05)$, sendo que os funcionários que possuem posições de chefia na organização atribuem médias estatisticamente superiores $(3,9177)$ a este fator comparativamente aos demais $(3,6747)$.

Da mesma forma que para o fator tempo de cargo, os resultados permitem inferir que os pesquisados que ocupam posições estratégicas na instituição estudada demonstram maior valorização pela busca e manutenção de relações sociais positivas no trabalho, bem como de gerar contribuições à sociedade mediante a realização de sua atividade. Nesse sentido, convém salientar a contribuição de Chanlat (2002), que enfatiza que a contribuição à sociedade é uma característica inerente ao setor público, e que trabalhar em beneficio da mesma serviria como motivação aos seus funcionários. Tais resultados são consistentes com 
os achados de Morin, Tonelli e Pliopas (2007), que afirmam que, no caso dos funcionários públicos, o sentido do trabalho pode assumir uma dimensão social, devido ao fato de servirem à sociedade.

\section{CONSIDERAÇÕES FINAIS}

Esse estudo desmistifica, em parte, o estereótipo do servidor público que valoriza apenas a segurança e a estabilidade, tendo em vista que os resultados demonstraram que esse profissional também almeja alcançar realização profissional.

Outro resultado importante refere-se à hierarquia de valores obtida neste estudo. Ao se considerar que os valores relativos ao trabalho são crenças acerca de comportamentos desejáveis, em que as diferentes metas são ordenadas por sua importância relativa às demais, evidenciou-se a seguinte hierarquia: Estabilidade e Realização no Trabalho, Relações Sociais e Prestígio. Ponderando que os valores, na estrutura proposta por Schwartz (1992, 1994), encontram-se dispostos em um continuum de importância, onde dimensões próximas denotam metas similares, a menor valorização do fator prestígio sinaliza para o fato de que, apesar dos pesquisados desejarem realização no trabalho, esses não buscam através de autoridade, sucesso profissional e poder de influência no trabalho.

No que tange às relações entre os fatores, observou-se que, na percepção dos funcionários pesquisados, a busca por prazer, realização pessoal e independência de pensamento e ação no trabalho está altamente correlacionada com a busca por relações sociais positivas por meio do trabalho.

Visando compreender as especificidades referentes às metas que indivíduos com características diferentes buscam alcançar com o trabalho, buscou-se compreender as diferentes percepções em relação às variáveis pessoais. Em relação ao gênero, os resultados propiciaram importantes contribuições acerca das metas motivacionais das mulheres.

Nesse sentido, observou-se que essas diferem em relação aos homens em dois fatores: Estabilidade e Realização no Trabalho, sendo que, em ambos, suas médias são superiores. Esse resultado remete a compreensão de que as mulheres diferem dos homens em relação à ênfase na busca por realização e estabilidade através do trabalho.

Convém salientar que, como enfatizam Silva, Mendonça e Zanini (2010), esses resultados podem estar relacionados ao papel desempenhado pela mulher na sociedade, passando, em muitos casos, a ser responsável pelo sustento familiar. Contudo, as funcionárias da instituição pesquisada, além de valorizarem estabilidade e segurança material, também desejam que a organização as propicie a realização de trabalhos desafiantes intelectualmente, nos quais possam ter autonomia e liberdade de pensamento e ação. Assim, o foco não está mais na busca de um trabalho que lhe propicie boas condições materiais e de segurança, mas, sobretudo, que traga também realização pessoal e profissional.

Em relação à idade, observou-se que os funcionários com idade superior à idade média buscam sucesso profissional e poder de influência no trabalho, concomitantemente com a manutenção de relações sociais positivas no trabalho (PORTO e TAMAYO, 2003). A busca por prestígio e manutenção de relações sociais positivas no trabalho por profissionais acima de 45 anos, dado o período de carreira que esses vivenciam pode estar relacionado a experiência e relações de trabalho já estabelecidas, as quais, de certa forma, influenciam a o contexto do trabalho, uma vez que estas relações sociais muitas vezes ultrapassam a fronteira organizacional, alcançando aspectos pessoais da vida do trabalhador. 
Em relação ao tempo de serviço, os resultados mostram que os pesquisados com tempo de cargo superior tendem a valorizar mais a busca de realização no trabalho. Tais resultados podem estar demonstrando que os profissionais pesquisados estão abertos à mudança em relação às novas oportunidades profissionais (MENDONÇA E TAMAYO, 2008), as quais podem ser até mesmo na própria instituição pesquisada. Cabe, enfim, a organização, de posse de tal entendimento, propiciar um ambiente desafiador e estimulante, no qual os funcionários realizem tarefas prazerosas.

Em relação ao tempo de cargo, os resultados demonstram que funcionários que estão há mais tempo no mesmo cargo tendem a maior valorização da busca e manutenção de relações sociais no ambiente de trabalho. Ainda em relação ao fator Relações Sociais, observou-se que esse também é o de maior valorização na percepção dos pesquisados que exercem cargos de chefia na Instituição.

Em relação a esse resultado, outro fato que merece destaque refere-se ao fato de a dimensão relações sociais apresenta interface com a dimensão de autotranscendência (SCHWARTZ, 1992; 1994). Tais resultados podem sinalizar que os funcionários com maior tempo de cargo e que possuem cargos de chefia, ao valorizarem relações sociais, desejam, de certa forma, contribuir para geração de benefícios sociais através de seu trabalho.

Um fator central nos resultados obtidos refere-se à valorização dos valores Realização no Trabalho, Estabilidade e Relações Sociais. Nesse sentido, acredita-se que as organizações deveriam direcionar seus esforços no sentido de desenvolver ações direcionadas a suprir tais metas motivacionais, uma vez que os valores relativos ao trabalho se encontram relacionados ao comprometimento, engajamento e motivação no trabalho (GOÉS, 2006; ROS, SCHWARTZ e SURKISS, 1999; PORTO e TAMAYO, 2003; LYONS, HIGGINS e DUXBURY, 2006; 2010).

Como limitações da pesquisa a impossibilidade de generalização dos resultados encontrados, visto que esses se relacionam ao estudo de uma realidade específica. Assim sendo, é importante considerar a necessidade de replicar o estudo com outras amostras e confrontar os dados aqui obtidos com outros setores públicos e outras instituições de Ensino Superior do país, bem como a realização de entrevistas em profundidade, a fim de enriquecer os resultados obtidos. Sugere-se a realização de estudos futuros que visem preencher esta lacuna, bem como incluir a análise dos valores organizacionais e pessoais.

\section{REFERÊNCIAS}

AAKER, D. A.; KUMAR, V.; DAY, G. S. Pesquisa de Marketing. São Paulo: Atlas, 2001.

ALMEIDA, F. J. R.; SOBRAL, F. J. B. A. O sistema de valores humanos de administradores brasileiros: adaptação da escala PVQ para o estudo de valores no Brasil. Revista de Administração Mackenzie, v. 10, n. 3, p. 101-126, 2009.

BILSKY, W. A estrutura de valores: sua estabilidade para além de instrumentos, teorias, idade e culturas. Revista de Administração Mackenzie, v. 10, n. 3, p. 12-33, 2009.

BRANDÃO, M.; BASTOS, A. V. B. Comprometimento organizacional em uma instituição universitária. Revista de Administração, v. 28, n. 3, p. 50-61, 1993.

CHANLAT, J. F. O gerencialismo e a ética do bem comum: a questão da motivação para o trabalho nos serviços públicos. In VII Congreso Internacional del CLAD sobre la Reforma del Estado y de la Administración Pública, Anais... Lisboa: 2002

GÓES. A. M. M. Valores relativos ao trabalho como antecedentes do comprometimento organizacional. 2006. $72 \mathrm{f}$. Dissertação (Mestrado em Psicologia) Universidade Católica de Brasília. Brasília, 2006.

HAIR JR., J., et al. Análise multivariada de dados. Porto Alegre: Bookman, 2009.

JING, J.; ROUNDS, J. Stability and change in work values: A meta-analysis of longitudinal studies. Journal of Vocational Behavior, v. xxx, n. xxx (In Press), 2011. 
LYONS, S. T.; HIGGINS, C. A.; DUXBURY, L. Work values: Development of a new three-dimensional structure based on confirmatory smallest space analysis. Journal of Organizational Behavior, v. 31, n. 7, p. 969-1002, 2010.

LYONS, S. T.; HIGGINS, C. A.; DUXBURY. L. A comparison of the values and commitment of private sector, public sector, and parapublic sector employees. Public Administration Review-Washington DC, v. 66, n. 4, 2006.

MALHOTRA, N. Pesquisa de marketing: uma orientação aplicada. Porto Alegre: Bookman, 2006.

MENDONÇA, H; TAMAYO, A. Valores e Retaliação Organizacional: estudo em uma organização pública. Revista de Administração Contemporânea-Eletrônica. v. 2, n. 2, p.189-200, 2008.

MORAES, L. F. R.; MARQUES, A. L.; CORREIA, L. F. Comprometimento organizacional: uma contribuição ao constructo. In: XXII ENCONTRO DA ASSOCIAÇÃO NACIONAL DOS PROGRAMAS DE PÓS GRADUAÇÃO EM ADMINISTRAÇÃO, 22, 1998. Foz do Iguaçu. Anais... Foz do Iguaçu: ANPAD, 1998.

MORIN, E. Os Sentidos do Trabalho. Revista de Administração de Empresas, v. 41, n. 3, p. 8-19, 2001.

MORIN, E.; TONELLI, M. J.; PLIOPAS, A. L. V. O Trabalho e Seus Sentidos. Psicologia \& Sociedade, v. 19, n. 1, p. 4756, 2007.

PESTANA, M.; GAGEIRO, J. Análise de dados para ciências sociais: a complementaridade do SPSS. Lisboa: Edições Sílabo, 2003.

PORTO, J. B.; TAMAYO, A. Valores do trabalho. In: SIQUEIRA, M. M. M. (Ed.). Medidas de comportamento organizacional: ferramentas de diagnóstico e de gestão. Porto Alegre: Artmed, 2008, p. 295-307.

PORTO, J. B.; TAMAYO, A. Estrutura dos valores pessoais: a relação entre valores gerais e laborais. Psicologia: Teoria e Pesquisa, v. 23, n. 1, p. 63-70, 2007.

PORTO, J. B. Mensuração de valores no Brasil. In: TAMAYO, A.; PORTO, J. (Ed.). Valores e comportamento nas organizações. Petrópolis: Vozes, 2005, p. 96-119.

PORTO, J. B.; TAMAYO, A. Escala de valores relativos ao trabalho - EVT. Psicologia: Teoria e Pesquisa, v. 19 n. 2 , p. 145-152, 2003.

ROCKEACH, M. The nature of human values. New York: Free Press, 1973.

ROE, R. A.; ESTER, P. Values and work: Empirical findings and theoretical perspective. Applied Psychology: An International Review, v. 48, p. 1-21, 1999.

ROHAN, M. J. A Rose by Any Name? The Values Construct. Personality and Social Psychology Review. v. 4, n. 3, p. 255-277, 2000.

ROS, M.; SCHWARTZ, S. H.; SURKISS, S. Basic individual values, work values, and the meaning of work. Applied Psychology: An International Review, v. 48, p. 49-71, 1999.

SCHWARTZ, S. Are there universal aspects in the structure and contents of human values? Journal of social issues, $v$. 50, p. 19-19, 1994.

SCHWARTZ, S. H. Universals in the content and structure of values: Theoretical advances and empirical tests in 20 countries. In: ZANNA, M. P. (Ed.). Advances in experimental social psychology. San Diego, CA: Academic, 1992.

SCHWARTZ, S. H. Valores humanos básicos: seu contexto e estrutura intercultural. In: TAMAYO, A.; PORTO, J. Valores e comportamento nas organizações. Petrópolis: Vozes, 2005.

SCHWARTZ, S. H.; BILSKY, W. Toward a universal structure of human values. Journal of Personality and Social Psychology, v. 53, n. 3, p. 550-562, 1987.

SILVA, M. R. M. S.; MENDONÇA, H.; ZANINI, D. S. Diferenças de Gênero e Valores relativos ao trabalho. Paidéia, v. 20, n. 45, 2010.

TAMAYO, A. Prioridades axiológicas, atividade física e estresse ocupacional. Revista de Administração Contemporânea, v. 5, n. 3, p. 127-147, 2001.

TAMAYO, A.; GONDIM, M. G. C. Escala de valores organizacionais. Revista de Administração da Universidade de São Paulo, v. 31, n. 2, p. 62-72, 1996.

TAMAYO, A.; MONIZ, A.; OLIVEIRA, V.; CARVALHO, R.; MACEDO, S.; ARMANDO, P.; GUIMARÄES, F. Prioridade axiológicas, tempo de serviço e cidadania organizacional; Value priorities, tenure and organizational citizenship. Psicologia: Teoria e Pesquisa, v. 14, n. 1, p. 35-40, 1998.

TAMAYO, A.; SCHWARTZ, S. Estrutura motivacional dos valores humanos. Psicologia: Teoria e Pesquisa, v. 9, n. 2, p. 329, 1993.

TAMAYO, M.; TRÓCCOLI, B. Exaustão emocional: relações com a percepção de suporte organizacional e com as estratégias de coping no trabalho. Estudos de Psicologia (Natal), v. 7, n. 1, p. 37-46, 2002. 